\title{
Communication de crise et maîtrise des risques dans les organisations
}

Arlette Bouzon

\section{OpenEdition}

1 Journals

Édition électronique

URL : http://journals.openedition.org/communicationorganisation/2257

DOI : 10.4000/communicationorganisation.2257

ISSN : 1775-3546

Éditeur

Presses universitaires de Bordeaux

Édition imprimée

Date de publication : 1 novembre 1999

ISSN : 1168-5549

Référence électronique

Arlette Bouzon, "Communication de crise et maîtrise des risques dans les organisations »,

Communication et organisation [En ligne], 16| 1999, mis en ligne le 27 mars 2012, consulté le 10

décembre 2020. URL : http://journals.openedition.org/communicationorganisation/2257 ; DOI :

https://doi.org/10.4000/communicationorganisation.2257

Ce document a été généré automatiquement le 10 décembre 2020.

(c) Presses universitaires de Bordeaux 


\title{
Communication de crise et maitrise des risques dans les organisations
}

\author{
Arlette Bouzon
}

1 Le 4 juin 1996, dans le ciel guyanais, explosait en vol le premier exemplaire d'Ariane 5, fleuron des ambitions européennes dans le domaine spatial. Face à cet événement, somme toute prévisible pour un prototype de cette nature, des millions de téléspectateurs restèrent incrédules. Ne leur avait-t-on pas répété des semaines durant que la principale caractéristique de ce lanceur était justement la fiabilité ? Devant les caméras, les responsables du programme semblaient décontenancés. Grisés par les succès d'Ariane 4, qui connut pourtant quelques déboires à ses débuts, ils n'imaginaient plus l'échec et n'avaient pas hésité à miser plusieurs milliards de francs en dotant la coiffe du lanceur de coûteux satellites scientifiques.

Outre des dysfonctionnements dans la gestion d'un programme technique à haut risque, cet exemple illustre les carences d'une politique de communication en période de crise.

Beaucoup d'organisations ne prennent en effet conscience du caractère stratégique de la communication que lorsque la crise survient. Elles tentent alors de se défendre par une improvisation brouillonne qui s'avère souvent désastreuse.

D'autres cherchent à se rendre moins vulnérables en élaborant des stratégies qui reposent le plus souvent sur des changements culturels au sein de leur personnel, afin de rendre l'organisation moins sujette à la critique ou plus à même de se défendre, ou sur des actions spécifiques ayant pour objet de mieux maîtriser les sources et les circuits d'informations diffusées vers l'extérieur.

5 Mais ces diverses stratégies mériteraient, nous semble-t-il, d'être associées à des techniques efficaces de gestion des risques qui ont déjà fait leurs preuves dans d'autres domaines. C'est pourquoi, après avoir montré combien crise et communication sont intimement liées et ne peuvent être traitées séparément, nous tenterons d'expliquer comment certaines méthodes et outils, utilisés par les spécialistes en Sûreté de Fonctionnement $(\mathrm{SdF})^{1}$ pour maîtriser les risques techniques, nous paraissent pouvoir s'appliquer à la prévention des crises et à la protection des organisations. 


\section{Crise et communication}

6 de la communication ont souligné le rôle souvent déterminant de la communication dans les situations de crise. C'est pourquoi nous envisageons ici la crise sous l'angle communicationnel.

Issue du terme grec Krisis signifiant « décision » c'est-à-dire une réponse à une situation particulière ${ }^{2}$, le terme «crise » a progressivement perdu son sens premier pour décrire aujourd'hui la situation elle-même.

8 Ainsi la crise est un moment décisif dans l'évolution d'un processus, qui s'apparente plutôt à l'indécision, tant la décision est souvent difficile à prendre en termes de choix et de mise en œuvre. En effet, si la crise constitue une menace qui met parfois en cause l'existence même de l'organisation, elle a souvent tendance à accroître les incertitudes.

9 La crise peut avoir pour origine une perturbation plus ou moins brusque, à l'intérieur ou à l'extérieur de l'organisation, ou résulter de phénomènes pernicieux difficilement perceptibles qui finissent par occasionner de graves dérèglements. L'après-crise se manifeste par l'accès à un nouvel équilibre : la crise étant bornée par deux périodes de stabilité relative.

10 Le terme de "crise» fait depuis quelques années l'objet d'un usage intensif ${ }^{3}$ pour expliquer des phénomènes divers, «au point que l'on peut se demander si sa généralisation n'a pas aussi pour fonction idéologique d'occulter des problématiques complexes et nouvelles » (Barus-Michel, Giust-Desprairies, Ridel, 1996, p. 12). Or, il n'existe pas une mais des crises. La réduction de leur diversité à une notion abstraite fait obstacle à leur compréhension et ne saurait rendre compte de la variété des processus engagés selon les unités sociales et les acteurs concernés.

11 La crise s'accompagne en général d'une crise de l'information, et qui ne maîtrise pas l'information ne maîtrise pas la crise ${ }^{4}$. En effet, l'information non contrôlée ou l'absence d'information augmente l'amplitude de la crise alors qu'une communication adaptée peut atténuer celle-ci (Lagadec, 1994). Ainsi, malgré un démarrage difficile, la communication qui accompagna l'échec du premier tir d'Ariane 5 sut atténuer son impact, et le demiéchec que rencontra le tir suivant passa pour un succès. Mais la maitrise de l'information recouvre l'usage qui en est fait car chaque média s'accapare de l'événement en lui donnant une tonalité particulière conformément au contrat de lecture (Maingueneau, 1996, p. 24) établi avec son public privilégié. E. Veron (1981) a ainsi montré comment les divers médias s'étaient appropriés l'accident de Three Mile Island qui affecta gravement une centrale nucléaire des États-Unis le 28 mars 1979.

12 La communication peut aider par la suite à sortir de la crise en proposant après coup une explication pseudo-rationnelle acceptable par tous, et en diffusant les enseignements qui en ont été retirés pour garantir l'avenir (Weick, 1993). Cette communication cherche avant tout à rassurer, à remotiver et à ressouder les membres d'une organisation qui a été fortement ébranlée. Elle permet de « reconstruire » en commun une représentation de la réalité, à partir de laquelle les individus peuvent se mobiliser à nouveau (Mucchielli, 1993, p. 77). Ainsi, bien que lié à une erreur de conception limitée à un sous-système particulier (la gestion à bord de la trajectoire), l'échec d'Ariane 5 fut considéré de responsabilité 
collective et donna lieu à des recommandations multiples adressées à tous les différents partenaires du projet.

Mais la communication peut être aussi un facteur de crise. Dans notre société de plus en plus médiatisée, une information mal maitrisée peut être elle-même source de crise, surtout quand elle est dirigée vers des partenaires de l'organisation particulièrement sensibles à l'image de celle-ci (clients, actionnaires, banquiers, syndicats...).

14 Ainsi, la chute extrêmement brutale du titre Alcatel, qui perdit plus de la moitié de sa valeur en septembre 1998, eut principalement pour origine la forme avec laquelle son PDG annonça des prévisions de résultats quelque peu décevants. Bien que ceux-ci ne furent pas catastrophiques, c'est l'impression d'opacité et de manipulation dégagée par cette action de communication qui fut fatale à l'entreprise et lui fit perdre la confiance d'investisseurs anglo-saxons habitués à plus de transparence.

L'information et la communication dans l'organisation peuvent également avoir des effets pathogènes (Mucchielli, 1993) et conduire à la crise. Celle-ci peut avoir pour origine des rumeurs non démenties (Kapferer, 1990a, 1990b) ou une communication paradoxale quand l'entreprise diffuse des messages contradictoires, simultanés ou échelonnés dans le temps, adressés à tout ou partie des acteurs internes ou externes (Watzlawick, HelminckBeavin, Jackson, 1979).

16 La crise peut également avoir pour origine la nature même de l'image de l'organisation, forgée par une communication efficace, qu'il est parfois difficile de renier quand elle s'avère, avec le temps, de plus en plus éloignée de la réalité. Ainsi peut-on expliquer l'« acharnement thérapeutique » qui a accompagné la fin du projet de la navette spatiale Hermès. Celui-ci, trop bien médiatisé, ne trouva personne dans les classes politiques, managériales et syndicales ayant le courage de proposer son arrêt alors que sa crédibilité technique et financière était entamée depuis bien des années. Cette incapacité à arrêter des projets de haute technologie fortement médiatisés mais aboutissant à des impasses est caractéristique d'une certaine élite technique, technocratique et politique française qui s'est encore illustrée dernièrement avec l'arrêt du surgénérateur de Creys-Malville dans la filière thermonucléaire de production d'électricité. Manifestation de carriérisme, peur du reniement ou force de l'image ? Le progrès scientifique et technique ne passe-t-il pas aussi par la reconnaissance du caractère erroné de certaines hypothèses?

Les interactions entre crise et communication sont multiples et nous n'avons envisagé que les plus courantes. Mais si crise et communication sont généralement indissociables, il importe cependant de communiquer dans l'urgence quand la crise survient.

\section{Communication de crise}

18 L'aspect médiatique de la crise, notamment quand celle-ci atteint son paroxysme, intéresse en premier lieu les responsables de la communication dans les organisations. En effet, outre l'exemple d'Ariane 5, l'actualité nous montre périodiquement combien les dirigeants d'entreprises sont souvent mal préparés à la crise et à la communication qui l'accompagne (Mucchielli, 1993). Certains optent indifféremment pour le silence complet, le mépris arrogant, les démentis en cascades, les déclarations trop rassurantes, le dégagement de toute responsabilité ou la mise en cause des journalistes et des médias... comportements qui ne peuvent qu'aggraver la situation. 
19 Pourtant l'enjeu est de taille et outre la survie des dirigeants, c'est parfois la pérennité même de l'entreprise qui est en jeu. Ainsi l'Union Carbide ne s'est jamais remise de l'accident de Bhopal et plus récemment, Mercedes-Benz a été fortement ébranlée par le retournement de ses petites voitures. Cette dernière organisation a su toutefois se rétablir en modifiant très rapidement ses produits et en menant une campagne de communication adaptée.

Si la communication en période de crise dépend énormément de la nature de celle-ci, le respect de quelques règles simples peut toutefois permettre de la désamorcer, ou du moins de ne pas l'amplifier. En l'absence de toute réaction, la crise prend de l'ampleur et il importe que l'organisation réagisse au plus vite pour ne pas laisser la situation devenir ingérable (Lagadec, 1991a, 1991b, 1994). Les dirigeants sont assaillis de demandes d'informations concernant l'événement et ses conséquences prévisibles, la désignation des responsables, et des engagements sur l'avenir... alors qu'un diagnostic complet n'est généralement pas encore établi. Il apparaît alors opportun d'exprimer une certaine compassion pour les victimes éventuelles et de tenter d'assurer leur prise en charge. Relayée par les médias, l'impression de mépris ou d'abandon peut, en effet, avoir un impact considérable. Il est aussi nécessaire de montrer que l'organisation met en œuvre tous les moyens dont elle dispose pour traiter correctement l'événement. Ce traitement peut recouvrir la recherche d'informations factuelles, la mise en œuvre de moyens de sauvegarde, la mise en place de commissions d'enquêtes crédibles, etc (Tixier, 1991). Dans cette phase difficile, il est souhaitable que la communication soit la plus transparente possible afin de ne pas pouvoir être prise en défaut et risquer de perdre toute crédibilité (Lagadec, 1994).

21 Ainsi, la communication de crise, qui est récemment devenue une véritable spécialité pour les professionnels, concerne l'ensemble des actions de communication qui accompagnent la crise que cela soit avant, pendant ou après celle-ci. Si des signes avantcoureurs permettent de prévoir la crise, la communication cherche à la désamorcer ou à la circonscrire dans une phase d'anticipation. Elle tente ensuite de la traiter à chaud pour ne pas lui laisser prendre une ampleur médiatique démesurée qui risquerait de déstabiliser l'organisation avant que celle-ci ne puisse réagir, ou de ternir définitivement son image. Elle accompagne enfin toutes les actions menées par l'organisation pour répondre à la crise puis pour en sortir dans les meilleures conditions.

L'« effondrement de sens » (Weick, 1993) ou " perte de l'univers de référence » (Lagadec, 1991a) qui caractérise chaque crise détermine le degré de gravité de la situation et les niveaux d'intervention nécessaires. La réponse communicationnelle sera différente selon que la crise concerne l'aspect opérationnel, quand elle est liée à des opérations de gestion courante, l'aspect stratégique, quand elle est provoquée par l'inadaptation, la nonlisibilité ou le rejet des objectifs de l'entreprise, ou l'aspect identitaire, quand elle révèle l'inadaptation de la mission ou des valeurs fondamentales de l'organisation (Plottu, 1998).

Différents ouvrages traitent de la communication de crise. Généralement prescriptifs et souvent illustrés d'exemples concrets, ils s'inscrivent dans un discours plus général mettant en évidence les vertus sociales de la communication dans des sociétés partiellement en déséquilibre ou en mutation (Dupont, Lachaud, 1998).

24 Cependant, outre la maîtrise des techniques et outils de communication, la meilleure réponse de l'organisation à la crise est toujours de l'éviter ou, si cela n'est pas possible, de bien s'y préparer. Or, peu d'écrits insistent sur ce point, même si certains auteurs 
proposent un audit de vulnérabilité pour mieux appréhender les faiblesses de l'organisation (Lagadec, 1991a, 1994 ; Robert, Verpeaux, 1991).

Pour notre part, il nous apparaît judicieux de reprendre la démarche générale de maîtrise des risques techniques utilisée par les spécialistes en Sûreté de Fonctionnement, que nous proposons d'appliquer à l'organisation et à son environnement en l'étendant notamment à l'aspect communicationnel.

\section{Vers une maîtrise des risques}

S'il apparait impossible d'éviter toutes les crises, l'organisation peut cependant chercher à limiter leur nombre et leur impact en suivant le processus général de maîtrise des risques utilisé par les fiabilistes pour se prémunir contre les risques techniques. Ce processus, en permanence sur l'entreprise et son environnement, peut être illustré par le schéma ${ }^{5}$ de la figure 1.

Ces risques, de nature technique, économique, humaine, sociale, organisationnelle, voire communicationnelle..., peuvent affecter l'entreprise ou son environnement, et sont liés à des événements aléatoires très divers. Mais le terme "événement" est riche, et «du même coup, il est polysémique, complexe, incertain, et il faut évidemment définir, distinguer, opposer les différentes variétés d'événements » (Morin, 1972, p. 176); ce que nous tenterons de faire ultérieurement.

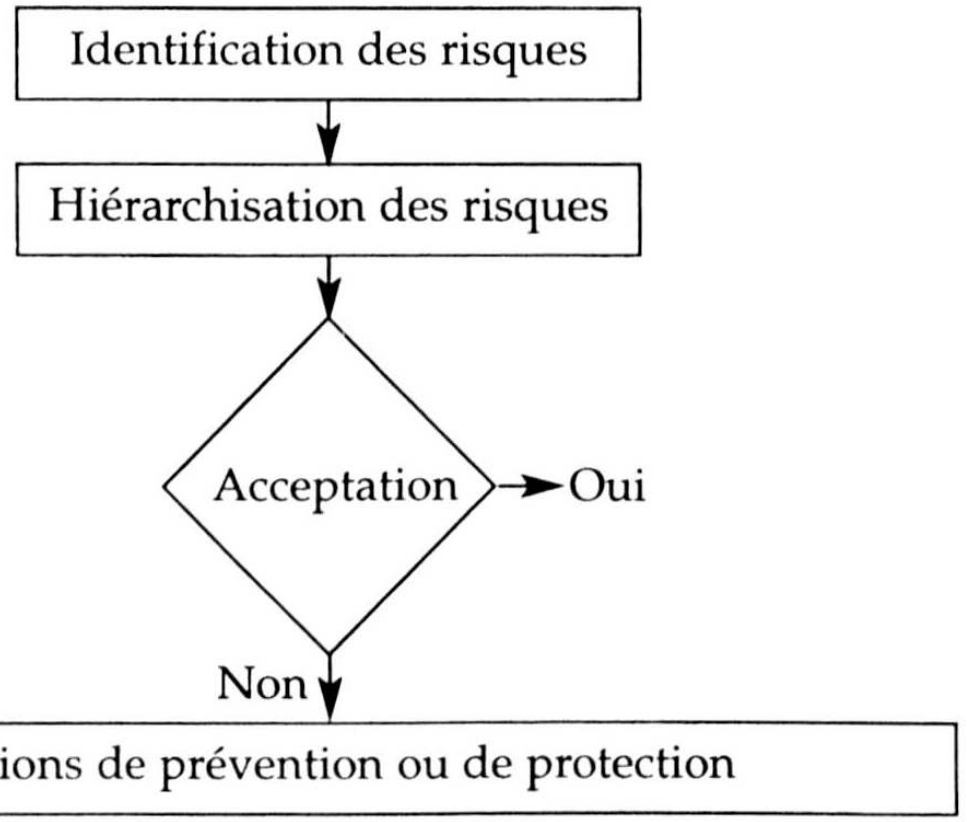

Figure $1:$ Processus de maîtrise des risques

La maîtrise des risques consiste à rechercher systématiquement les risques auxquels l'entreprise est confrontée, les hiérarchiser suivant leur gravité et leur probabilité d'occurrence, puis les accepter ou les traiter. Ce traitement consiste à mettre en œuvre des actions de prévention, en intervenant sur les causes possibles des événements redoutés afin d'éviter qu'ils ne surviennent (mise en place de panneaux d'interdiction de fumer, par exemple), ou de protection pour en diminuer les effets (installation 
d'extincteurs et affichage de plans d'évacuation). Ces mesures sont d'autant plus efficaces qu'elles sont prises suffisamment à l'avance car, d'une part, la crise entraîne une progression des incertitudes, et donc une régression de la prédiction (Morin, 1972, 1976), et, d'autre part, les actions en réduction de risques sont parfois longues à mettre en œuvre.

La définition préalable d'un domaine d'acceptabilité propre à l'organisation concernée, tel que celui décrit à la figure 2 (Pages A., Gondran M., 1980), permet d'accepter ou de rejeter chacun des risques identifiés.

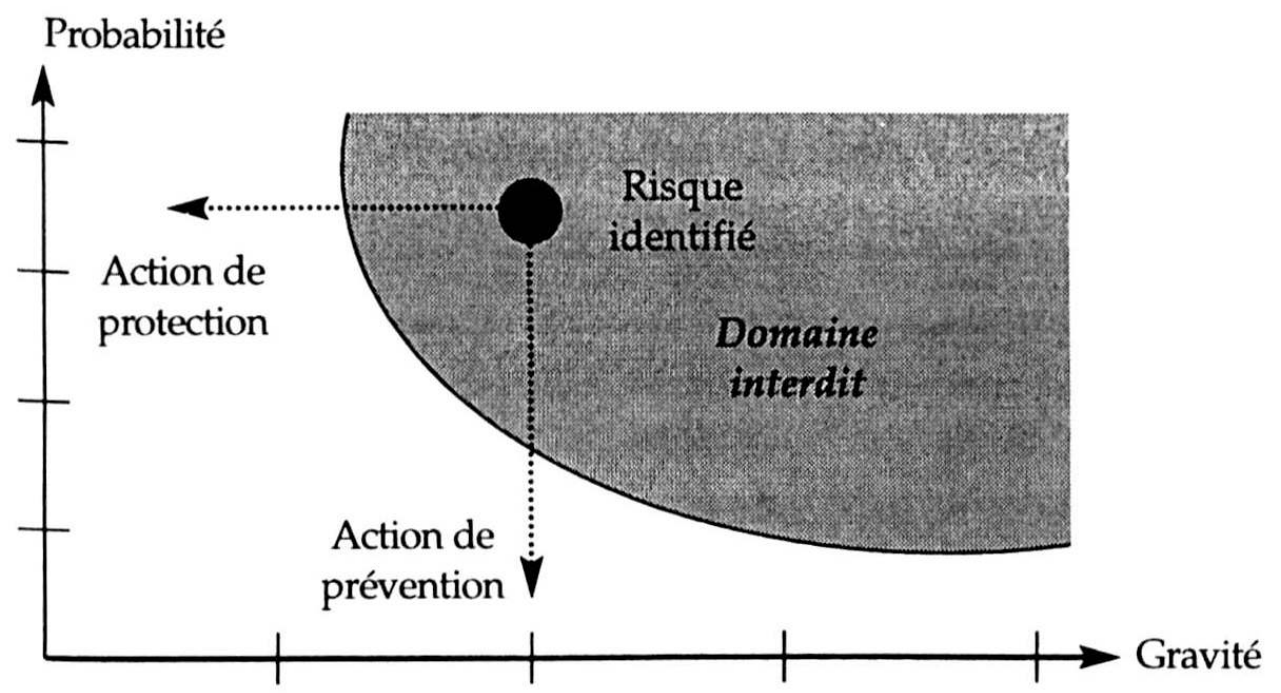

Figure 2 : Domaine d'acceptabilité des risques

Pour faciliter ce traitement, les variables de gravité et de probabilité concernées font généralement l'objet d'une classification, qui, dans le cas qui nous intéresse, pourrait être la suivante (figure 3 ) :

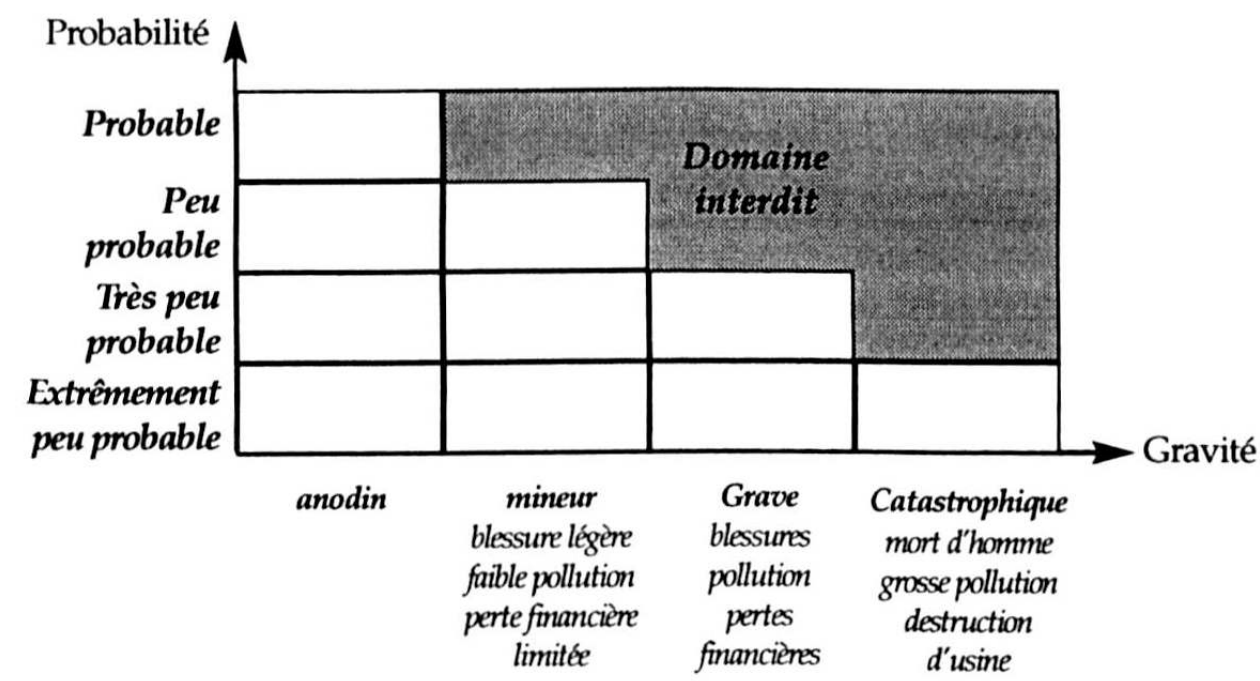

Figure 3 : Exemple de grille d'acceptabilité

L'identification des risques peut résulter de l'exploitation du retour d'expérience (capitalisation de l'expertise et des enseignements passés), d'une démarche deductive 
consistant à imaginer les causes possibles de situations critiques, ou d'une démarche inductive cherchant à évaluer les effets sur l'organisation de dysfonctionnements élémentaires (Pages A., Gondran M., 1980 ; Villemeur, 1987).

L'expérience des organisations est souvent mal exploitée bien que nous assistions aujourd'hui à une prise de conscience de son caractère stratégique par de nombreux dirigeants d'entreprise. Elle est généralement difficile à formaliser et apparaît au détour de multiples notes, règlements, rapports d'incident et documents divers. Elle réside même parfois dans les seules connaissances et savoir-faire de quelques individus qui les divulguent ou les transmettent à leur gré de manière partielle et épisodique. Cette expérience est souvent biaisée pour des raisons diverses. Certains membres de l'organisation sont tentés, consciemment ou non, de la garder pour eux-mêmes ou de ne la divulguer qu'en partie, afin de garder le pouvoir qu'elle procure (Crozier, Friedberg, 1977). Les rapports d'incident et les recommandations qui les accompagnent ne donnent pas toujours une vision objective des événements considérés, car ils cherchent souvent à donner des justifications a posteriori de situations anormales afin de préserver tout ou partie de l'organisation (Lagadec, 1991, p. 302).

Pour concourir efficacement à la maîtrise des risques, cette expérience peut être formalisée au moyen de règles et procédures consignées dans les spécifications, manuels de qualité et règlements de l'entreprise dont le respect permet d'éliminer a priori certains risques. Elle peut également se matérialiser sous la forme de listes de risques génériques, que l'on balaie périodiquement ou lors d'événements particuliers en les employant comme de véritables « check-lists " ${ }^{6}$.

La recherche déductive des causes possibles d'événements redoutés ${ }^{7}$ peut concerner l'organisation dans son ensemble (missions, objectifs, structures...) ou une partie de celleci (services, projets, produits, organisations syndicales...). Afin d'être efficace, l'imagination de l'analyste ne doit pas être bridée et doit pouvoir, si nécessaire, briser certains tabous. En effet, l'analyse a posteriori d'accidents graves, relatés au travers des rapports de commission d'enquêtes, montre le plus souvent des comportements irrationnels, des impasses incompréhensibles, des inadaptations criantes ou des situations aberrantes...

La recherche déductive s'intéresse également à l'environnement de l'organisation. En raison de l'évolution de plus en plus rapide des produits et des technologies, l'entreprise doit se tenir informée en permanence des modifications de son environnement extérieur pour ne pas risquer de se trouver brutalement en dehors du marché. Cette veille technologique et stratégique, appelée aujourd'hui intelligence économique (Rouach, 1996), concerne aussi bien les produits développés par la concurrence, que les technologies mises en œuvre, ou les méthodes utilisées. Elle ne peut plus exclure la recherche scientifique dont les retombées pratiques sont parfois radicales et souvent beaucoup plus rapides que par le passé. Cette veille a longtemps été assumée par les spécialistes de l'organisation en plus de leurs activités propres, mais la masse d'informations à traiter ne permet plus ce mode de fonctionnement et la plupart des grandes entreprises se dotent aujourd'hui de services spécialisés. Ceux-ci sont aidés dans leurs recherches par des moyens divers (internet, moteurs de recherche, cabinets spécialisés...) et sont en contact direct avec les spécialistes de l'organisation pour définir les axes de recherches et évaluer les informations recueillies. 
36 La bonne connaissance de l'actionnariat de l'entreprise permet d'assurer sur lui un minimum de contrôle et pallier certains risques majeurs souvent négligés tels que la désaffection brutale d'investisseurs volatiles ou le soudain intérêt d'organisations étrangères cherchant à prendre le contrôle de l'entreprise par de massifs achats d'actions.

37 Le suivi et l'analyse des grands mouvements d'opinion participent également à la maitrise des risques auxquels l'entreprise est confrontée, non seulement parce qu'ils influencent fortement les attentes des éventuels clients, mais aussi parce qu'ils peuvent rendre insupportables certaines pratiques courantes longtemps tolérées. Ces dernières peuvent par exemple concerner la préservation de l'environnement, la sécurité des consommateurs ou des citoyens, ou des règles d'éthique telles que la lutte contre la corruption ou le respect des droits de l'homme.

38 Assimilable à l'AMDEC (Analyse des Modes de Défaillance, de leurs Effets et de leurs Criticités) utilisée par les fiabilistes pour appréhender les effets de certaines défaillances, la recherche inductive des effets de chacun des dysfonctionnements possibles dans l'entreprise apparaît relativement lourde et difficilement applicable aux multiples rouages d'une organisation. Elle peut cependant porter sur les événements marquants prévus dans le calendrier de l'entreprise, afin d'évaluer pour chacun l'impact d'un déroulement non conforme aux attentes, ou sur certains événements plus ou moins prévisibles dont l'occurrence peut être annoncée par des signes avant-coureurs. Les événements susceptibles d'être ainsi traités pourraient être regroupés de la manière suivante :

39 - Événements bien identifiés : tests et essais de nature technique, passage à l'an 2000 (problème d'informatique), passage à l'Euro, lancement d'un nouveau produit, campagne de communication, communication aux actionnaires, opérations de fusion ou de rachat, introductions en bourse...

40 - Événements prévisibles à date indéterminée : retournements de conjoncture, réveil de la concurrence après le lancement d'un produit, modification des règles concurrentielles (fin de situation monopolistique)...

41 - Événements aléatoires : grèves, O.P.A., accidents...

42 L'identification des risques de la première catégorie est relativement simple car elle consiste à envisager l'échec ou des difficultés concernant des événements ou actions prévus à des moments bien déterminés. Cette aptitude à imaginer le pire, que l'habitude et l'excès de confiance en soi finissent parfois par annihiler, conduit à être prêt à toute éventualité. Ainsi, il n'est pas nécessaire de communiquer bruyamment en faisant preuve d'un excès d'optimisme avant chaque événement à risques, ce qui ne fait que renforcer l'incrédulité et la déception en cas d'échec. Par ailleurs, le discours accompagnant cet événement peut être préparé à l'avance suivant les divers scénarios possibles.

43 Les risques liés à des événements prévisibles mais à des moments indéterminés peuvent être également traités par ce type d'analyse. Mais beaucoup d'organisations font preuve d'une grande cécité et n'ont parfois aucune capacité d'anticipation. Apparemment efficaces dans un environnement stable, elles se révèlent incapables de faire face au changement. Ainsi beaucoup d'entreprises ne purent envisager la chute du dollar après son envolée durant les premières années du libéralisme reaganien, puis presque toutes les banques se laissèrent surprendre par la crise de l'immobilier au début des années quatre- 
vingt-dix, et combien d'organisations ont pu imaginer la catastrophe financière qui s'est abattue récemment sur l'Asie du sud-est?

Cette observation est à rapprocher des conclusions de diverses études sur le «facteur humain» menées par des ergonomes dans le cadre de commissions d'enquêtes (catastrophe aérienne, accident nucléaire, etc.) qui montrent que face à un système complexe l'homme se construit mentalement un modèle théorique plus ou moins précis. Le management de l'entreprise conduit à la construction d'un tel modèle de l'organisation et de son environnement, mais, si celui-ci est en général suffisant en période de croissance économique, c'est sa validité qui conditionne la disparition ou la survie de l'organisation quand la conjoncture évolue. La culture des individus joue un grand rôle dans l'émergence et les usages de ces modèles, mais au lieu de tenter d'aller à rencontre de cette "tendance naturelle", l'entreprise peut juger préférable de s'en servir. Ainsi un pilote d'essai ne percevra pas une situation opérationnelle de la même manière que le technicien qui a construit son avion. Le but n'est pas de transformer un technicien en pilote, ni inversement, mais de se servir des éléments apportés par la culture de chacun pour mieux appréhender cette situation. C'est pourquoi il est souvent bénéfique pour l'organisation que les décideurs puissent considérer des points de vue différents, voire contradictoires, émis par des personnalités d'horizons divers.

Parmi les actions mises en œuvre par l'organisation pour ne pas être affectée par de tels événements, la communication peut être utilisée pour préparer l'opinion aux risques encourus et désamorcer ainsi les crises avant qu'elles ne surviennent. Elle peut être également dirigée vers les publics internes de l'entreprise pour les sensibiliser et les mettre en alerte.

Les risques liés à des événements aléatoires sont, par définition, plus difficiles à prévoir et peuvent surtout se traiter par des actions palliatives telles que l'instauration de mécanismes d'alertes précoces, l'établissement de procédures d'urgence pour améliorer la réactivité de l'entreprise (mise en place de cellules de crise regroupant des personnes susceptibles de se réunir rapidement et de prendre des décisions...), ou le déploiement de moyens de sauvegarde pour limiter les effets d'une crise éventuelle (moyens de sécurité, assurance, coalition de défense entre partenaires...). La communication interne intervient évidemment dans ces diverses actions pour sensibiliser les membres du personnel, assurer leur entraînement régulier aux procédures de crises et tester ces dernières par la même occasion.

47 Et si malgré tout la crise survient, l'après-crise constitue un moment particulièrement important durant lequel l'entreprise peut capitaliser une expérience utile en vue de prévenir le renouvellement de situations similaires et améliorer les procédures mises en place.

\section{Conclusion}

Au-delà des techniques communicationnelles habituellement proposées pour gérer les crises, cet article présente succinctement des méthodes susceptibles d'éviter l'apparition de celles-ci ou d'en amoindrir les effets. Même si l'incertitude ne peut être complètement maîtrisée, il apparaît en effet possible de se prémunir d'une grande partie des risques auxquels l'organisation est soumise, en appliquant à l'entreprise et à son environnement les méthodes mises en œuvre par les fiabilistes pour traiter les risques techniques. Ces 
méthodes concourent à la maîtrise des risques en cherchant tout d'abord à les identifier, puis à les évaluer avant de prendre les mesures adéquates de prévention ou de protection, dont éventuellement certaines actions d'information et de communication.

Il faut souligner que cette maîtrise des risques ne peut pas être assurée par l'utilisation de recettes génériques, concernant notamment la communication, mais passe par l'emploi d'une démarche globale et continue conduisant à des actions spécifiques qui dépendent des situations et des risques identifiés.

Mais cette approche rationnelle se heurte à de multiples freins culturels dans les organisations. Celle-ci suppose, en effet, un regard critique le plus objectif possible sur l'entreprise et son fonctionnement afin d'en appréhender les faiblesses, puis de remédier à celles-ci par des actions qui remettent souvent en cause le jeu des pouvoirs établis.

Elle nécessite un engagement des dirigeants, une forte sensibilisation du personnel et une responsabilisation globale de l'organisation. Celle-ci se traduit par de nombreuses délégations de pouvoir et une responsabilisation accrue du personnel à tous les niveaux. Elle implique de perdre de mauvaises habitudes, qu'elles soient personnelles (rétention d'information, déchargement systématique sur d'autres des problèmes rencontrés sans se soucier réellement de leur prise en charge, fermeture et incompréhension...), ou organisationnelles (flou et ambiguïté des responsabilités, cloisonnement, retour d'expérience inexistant...). Elle remet en cause certaines idées qui s'avèrent généralement fausses, telles que le coût démesuré des actions de prévention ou de protection des risques les plus critiques. Elle peut conduire à modifier certains objectifs de l'organisation ou adopter certaines règles d'éthique afin de ne pas transiger avec des risques d'une certaine gravité (sécurité, environnement, corruption...).

Elle demande enfin un effort de formation important à la maitrise des risques pour tout le personnel en place et à la communication en situation de crise pour l'ensemble des dirigeants.

53 Ainsi le problème n'est pas tellement de bien communiquer en période de crise mais plutôt d'éviter la crise et si, malgré tout, celle-ci survient, d'avoir quelque chose de crédible à communiquer. La communication sert alors à montrer la bonne foi de l'organisation qui a tout fait pour éviter la crise et qui fera tout son possible pour la surmonter.

54 Cette approche impose à l'organisation d'être en phase avec son environnement, d'assumer ses responsabilités et de ne plus chercher à avoir raison... seule contre tous. Le nuage de Tchernobyl, la « vache folle » et l'affaire du sang contaminé ont en effet marqué la fin d'une période où des certitudes techniques pouvaient être assénées par des autorités incontestables.

55 Mais en matière de crise, la vigilance reste de rigueur car quelle que soit la panoplie de règles ou d'outils utilisés, le risque demeure. La démarche que nous proposons ici nous semble pouvoir le réduire même si elle mériterait certains approfondissements. 


\section{BIBLIOGRAPHIE}

Barus Michel J., Giust-Desprairies F, Ridel L., Crises. Approche psychosociale clinique, Paris, Desclée de Brouwer, 1996, 315 p.

BELL R., Les péchés capitaux de la haute-technologie, Paris, Seuil, 1998, 345 p.

CROzIER M., Friedberg E., L'acteur et le système, les contraintes de l'action collective, Paris, Seuil, 1977, $436 \mathrm{p}$.

DogAn M., PAHRE R., L'innovation dans les sciences sociales. La marginalité créatrice, Paris, PUF, 1991, $322 \mathrm{p}$.

DUPONT O., LACHAUD G., « L'acteur, le groupe et l'institution. Dynamique individuelle et collective de l'urgence ", Sciences de la société, 1998, n 44, pp. 44-62.

HAUCH V., « Paradoxe et crise dans l'organisation : une interprétation en terme de double contrainte ", in colloque Urgences, gestion des crises et décision, Tarbes, 1997, 17 p., polycopié.

HERMANN $\mathrm{CF}_{\mathrm{F}}$., "Some consequences of crisis which limit the viability of organizations ", Administrative Science Quaterly, 1963, 8, pp. 61-82.

KAPFERER J.N., Rumeurs : le plus vieux média du monde, Paris, Le Seuil, 1987, 240 p.

KAPFERER J.N., « Le contrôle des rumeurs », Communications, 1990 (a), 52, pp. 99-117.

KAPFERER J.N., « La rumeur en bourse », Communications, 1990 (b), 52, pp. 61-84.

LAGADEC P., La gestion des crises, Paris, Me Graw Hill, 1991 (a), 323 p.

LAGADEC P., « Pourquoi et comment bâtir un plan de communication préventive » in TIXIER M., La communication de crise, Paris, Mc Graw Hill, 1991 (b), pp. 81-103.

LAGADEC P., Apprendre à gérer les crises, Paris, Les éditions d'organisation, 1994, 120 p.

LAULHERET R., Maîtrise des risques techniques, in Cours de technologies spatiales, Toulouse Édition Cepadues, 1998, pp. 320-362.

Maingueneau D., Les termes clés de l'analyse de discours, Paris, Seuil, 1996, 94 p.

MORIN E., « L'événement-sphinx », Communications, 1972, vol. 18, pp. 173-192.

MoRIN E., « Pour une crisologie », Communications, 1976, vol. 25, pp. 149-163.

Mucchielli A., Communication interne et management de crise, Paris, Les éditions d'organisation, 1993, $207 \mathrm{p}$.

OGRIZEK M., GUILLERY J.M., La communication de crise, Paris, Puf, 1997,128 p.

PAGES A., Gondran M., Fiabilité des systèmes, Paris, Édition Eyrolles, 1980, 348 p.

Plottu E., « Les crises et leur gestion », Sciences de la société, 1998, nº 44, pp. 145-164.

REVERET R., MOREAU J.N., Les médias et la communication de crise, Paris, Economica, 1997, 112 p.

ROBERT B., VeRPEAUX D., « Pourquoi et comment bâtir un plan de communication préventive », in TIXIER M., (dir.), La communication de crise, Paris, Mc Graw Hill, 1991, pp. 81-103. 
RouACH D., La veille technologique et l'intelligence économique, Paris, Puf, 1996,128 p.

ROUX-DUFORT C, « Apprendre des crises. Entre le statu quo et la transformation », Sciences de la société, $1998, \mathrm{n}^{\circ} 44$, pp. 165-182.

RUFFIER J., « Fonctionnement normal, fonctionnement critique. Les situations industrielles hautement risquées ", Sciences de la société, 1998, nº 44, pp. 113-145.

Tном R., Esquisse d'une sémiophysique, Paris, InterEditions, 1988, 285 p.

TIXIER M., (dir.), La communication de crise, Paris, Mc Graw-Hill, 1991, 268 p.

WATZLAWick P, HeLMinCK-BEAVIN J., JACKSON D., Une logique de la communication, Paris, Seuil, 1979, $288 \mathrm{p}$.

WEICK K.E., ROBERTS K.H., « Collective mind in organizations, Heedful interrelating on Flight Decks », Administrative Science Quaterly, vol. 38,1993, pp. 359-383.

WEICK K.E., « The non-traditional quality of organizational learning », Organizational Science, vol. 2, 1991, pp. 116-123.

VERON E., Construire l'événement. Les médias et l'accident de three mile island. Paris, Éditions de minuit, 1981, $177 \mathrm{p}$.

VILlemeur A., Sûreté de Fonctionnement des systèmes industriels, Paris, Édition Eyrolles, 1987, 795 p.

\section{NOTES}

1. La Sûreté de Fonctionnement est une activité d'ingénierie souvent intégrée à la Qualité qui cherche à améliorer la fiabilité, la disponibilité et la sécurité des produits et des processus de fabrication. Elle tente de maîtriser les risques techniques Liés à différents aléas (défaillances, erreurs humaines, environnement anormal...).

2. Rey A. (dir.). Dictionnaire historique de la langue française, Paris, Dictionnaire le Robert, 1995, p. 530.

3. Dérive stigmatisée par de nombreux auteurs (Morin E., 1976 ; Thom R., 1988 ; Barus-Michel, Giust-Desprairies, Ridel, 1996...).

4. La crise génère également de l'information sur ta vulnérabilité de l'organisation et sa capacité à réagir.

5. Schéma souvent utilisé dans les ouvrages spécialisés en SdF (Pages A., Gondran M., 1980 ; Laulheret R, 1998...).

6. De telles «check-lists" sont notamment utilisées en matière de sécurité pour identifier des risques affectant des personnes ou des biens (incendie, explosion, intoxication, pollution...), mais peuvent également concerner tout autre type de risques.

7. À partir d'un événement redouté, cette recherche s'effectue généralement au moyen d'un arbre d'événements logiques que nous ne développons pas dans cet article. 


\section{RÉSUMÉS}

Après avoir montré comment certaines organisations peuvent être fragilisées par une communication inadaptée à des moments critiques de leur histoire, cet article souligne les apports de la communication de crise pour surmonter celle-ci puis pour aider à en sortir. Mais ces méthodes de communication apparaissent limitées en regard des enjeux, si elles ne sont pas associées à des techniques efficaces de gestion des risques. C'est pourquoi nous proposons que certaines méthodes et outils, utilisés par les spécialistes en Sûreté de Fonctionnement pour maîtriser les risques techniques, soient adaptés à la prévention des crises et à la protection des organisations.

After showing how helpless some organizations are when confronted to a crisis and how shaken they can be by an inadequate communication plan, this article stresses the contribution of crisis communication in both overcoming such crucial junctures and then contriving a favorable issue. But these communication methods seem limited compared with the stakes if they are not used in conjunction with effective management techniques. This is the reason why we consider that some tools and methods used by Operating Reliability specialists to control technical risks are well adapted to prevent crises and to protect organizations.

\section{AUTEUR}

\section{ARLETTE BOUZON}

Ariette Bouzon est maître de conférences en sciences de la communication à l'Université Paul Sabatier-Toulouse III et responsable de la formation en communication d'entreprise de l'ITUT information/communication (UPS-Toulouse III). Chercheur au Lerass, membre de l'équipe Information et communication entre chercheurs (I.C.C.) elle est aussi membre du groupe d'études et de recherches sur les communications organisationnelles (Org and Co) de la SFSIC. 